\title{
THEORETICAL STUDIES OF POLARIZATION DEPENDENT ELECTRO-OPTICAL MODULATION IN LATTICE MATCHED AND STRAINED MULTI-QUANTUM WELL STRUCIURES
}

\author{
Songcheol Hong and Jasprit Singh \\ Center for High Frequency Microelectronics \\ Department of Electrical Engineering and Computer Science \\ The University of Michigan \\ Ann Arbor, Michigan 48109
}

(Received 17 August 1987)

\begin{abstract}
We report on polarization dependent optical absorption for excitonic and interband transitions in lattice matched (GaAs/AlGaAs) and strained (biaxial tensile strain $\mathrm{GaAsP} / \mathrm{AlGa \Lambda s}$; biaxial compressive strain - InGaAs/AlGaAs) multiquantum well structures in the presence of transverse electric fields. The hole states are solved by using the Kohn-Luttinger Hamiltonian and using an eigenvalue technique. The effect of heavy-hole and light-hole mixing due to the strain, electric field and quantization is studied. Under biaxial tensile strain the heavy-hole and light-hole transition can coincide, leading to interesting polarization dependent effects. Results are presented for excitonic and interband transitions.
\end{abstract}

\section{INTRODUCTION}

Optical absorption in multiquantum well (MQW) structures has become an important area of research due to potential applications in optical devices as well as a probe to study new physics in reduced dimensions. ${ }^{2}$ Exciton transitions in MQW structures in particular are acquiring special importance due to the fact that the enhanced exciton binding energy and presence of the barrier region allows excitonic transitions to persist upto high temperatures and at high transverse electric fields. ${ }^{2,3}$ This has led to a number of structures which have applications as optical modulators, optical switches, optical couplers etc. ${ }^{4,5}$ Devices based on both vertical light incidence and wave guide geometries have been proposed and demonstrated. ${ }^{6.21}$ In the wave guide geometry (light incident parallel to the interfaces of the quantum well) strong polarization dependences of the absorption and emission processes are observed. This polarization dependence has potential applications in uptical processing technology and also as a probe to study important band structure properties.

In this paper we will discuss the theoretical basis for the polarization dependence of optical absorption in lattice matched and strained MQW structures. The interest in strained layer systems arises because by appro- priate choice of strain sign(i.e. biaxial tensile or compressive) in the well region, the heavy-hole( $\mathrm{HH})$ and light-hole(LH) transitions can be merged or separated in energy. The strain can thus control the HH-LH band mixing and lead to interesting polarization dependences of the absorption. This study is carried out by using the Kohn-Luttinger Hamiltonian to describe the $\mathrm{HH}$ and $\mathrm{LH}$ states and their interaction in both lattice matched and strained systems. Polarization dependences of both excitonic and band-to-band transitions are then calculated in the absence and presence of a transverse electric field. In the next section we describe the formalism used for the calculations. In section III,the results are presented. Discussion and conclusions are presented in section IV.

\section{THEORETICAL FORMALISMS}

In this section we will discuss the theoretical techniques used by us to obtain the results presented in this paper. The optical absorption problem in a strained quantum well can be divided into several key components viz. i) the electron(e) and hole (h) problem in a quantum well, $i i)$ the effect of strain on the $e, h$ problem in a quantum well, iii) effect of transverse electric field, 
iv) the excitonic problem and finally, v) the optical absorption and its polarization dependence. We will now discuss each of these components.

i) Electron and hole problem in quantum wells:

To solve the exciton problem in quantum wells one needs to understand the in-plane (perpendicular to the growth direction taken as the (001) direction for the cases studied here) band dispersion relations for the electron and the hole problem. The solution of this problem is simplied if we assume that the electron states are nondegenerate (except for spin) and originate from $\mid s>$ like atomic functions while the hole states are $\mid p>$ type and are 4-fold degenerate (including the spin degeneracy). Such an approximation is quite valid for most III-V compound semiconductors. In presence of an arbitrary quantum well the electron problem can be simply written as:

$$
-\frac{\hbar^{2}}{2 m} \nabla^{2} \Psi(\mathbf{k}, z)+V(z) \Psi(\mathbf{k}, z)=E \Psi(\mathbf{k}, z)
$$

where $V(z)$ represents the potential barrier seen by the electrons. In the direction parallel to the z-axis, the momentum vector $\mathbf{k}$ is a good quantum number and the solution has a general form

$$
\Psi_{e l}^{n}(\mathbf{k}, z)=f^{n}(z) U_{0}(r) e^{i \mathbf{k}_{\|} \cdot \rho}
$$

where $f^{n}(z)$ are the electron envelope functions for the 1-dimensional potential well problem and $U_{0}(r)$ is the zone-center conduction band Bloch function for the bulk well material. The electron envelope function can be solved by variational techniques or other standard numerical techniques for solving differential equations.

The hole states are much more complicated to solve because of the four-fold degeneracy at the zone center. We use the Kohn-Luttinger Hamiltonian, as given in equation (3), for the lattice matched system: ${ }^{7}$

$$
\left[\begin{array}{cccc}
H_{h h}+\frac{\delta}{2} & c & b & 0 \\
c^{*} & H_{l h}-\frac{\delta}{2} & 0 & -b \\
b^{*} & 0 & H_{l h}-\frac{\delta}{2} & c \\
0 & -b^{*} & c^{*} & H_{h h}+\frac{\delta}{2}
\end{array}\right]\left[\begin{array}{c}
H \Psi= \\
\Phi_{\frac{3}{2} \frac{3}{2}} \\
\Phi_{\frac{3}{2} \frac{1}{2}} \\
\Phi_{\frac{3}{2} \frac{-1}{2}} \\
\Phi_{\frac{3}{2} \frac{-3}{2}}
\end{array}\right]
$$

where $\delta$ is the separation of $\mathrm{HH}$ and LH states in a bulk material due to the strain. ${ }^{8}$

Treating the $z$-component of the momentum as an operator $\left(k_{z}=-i \frac{\partial}{\partial z}\right)$ we get the following new expressions for the matrix elements :

$$
\begin{aligned}
H_{h h}= & -\frac{\hbar^{2}}{2 m_{\circ}}\left[\left(k_{x}^{2}+k_{y}^{2}\right)\left(\gamma_{1}+\gamma_{2}\right)-\left(\gamma_{1}-2 \gamma_{2}\right) \frac{\partial^{2}}{\partial^{2} z}\right] \\
& +V_{p}(z)
\end{aligned}
$$

$$
\begin{aligned}
H_{l h}= & -\frac{\hbar^{2}}{2 m_{o}}\left[\left(k_{x}^{2}+k_{y}^{2}\right)\left(\gamma_{1}-\gamma_{2}\right)-\left(\gamma_{1}+2 \gamma_{2}\right) \frac{\partial^{2}}{\partial^{2} z}\right] \\
& +V_{p}(z) \\
c= & \frac{\sqrt{3} \hbar^{2}}{2 m_{o}}\left[\gamma_{2}\left(k_{x}^{2}-k_{y}^{2}\right)-2 i \gamma_{3} k_{x} k_{y}\right] \\
b= & \frac{\sqrt{3} \hbar^{2}}{m_{o}}\left(-k_{y}-i k_{x}\right) \gamma_{3} \frac{\partial}{\partial z}
\end{aligned}
$$

where $\gamma_{1}, \gamma_{2}, \gamma_{3}$ are the Kohn-Luttinger parameters. As shown in eq. 3 , HH and LH band are degenerate at zone center in bulk material with out strain, but with quantum well potential they are no longer degenerate because of their different effective masses. The strain provides an increase or decrease in the splitting of $\mathrm{HH}$ and $\mathrm{LH}$ bands which is depend on the direction of strain.

The general hole solutions can be written as

$$
\Psi_{h}^{m}(\mathbf{k}, z)=\sum_{\nu} g_{m}^{\nu}(z) U_{v}^{\nu}(r) e^{i \mathbf{k}_{\|} \cdot \rho}
$$

where $g_{m}^{\nu}(z)$ is the $z$-dependent function arising from the confinement of the potential, $\nu$ is the index representing the total angular mommentum of the state, $m$ is the index for the each subband in the well and $V_{0}^{\nu}(r)$ is the zone-center valance-band Bloch state for the $\nu$ spin component in the bulk material.

In the absence of the off-diagonal mixing terms in the Kohn-Luttinger Hamiltonian, (the so called diagonal approximation) the hole problem is as simple to solve as the electron problem. However, in real semiconductors the off diagonal mixing is quite strong and must be included for quantitative comparison with experiments. The off-diagonal terms are also responsible for occurence of the normally "forbidden" transitions".

The Schrodinger equation, using the Kohn Luttinger Hamiltonian, is written as a finite difference equation which can then be solved by standard eigenvalue methods. This yields the hole dispersion relations in the quantum well. The dispersion relations in strained quantum wells can also be accurately delemmined using the modified Kohn-Luttinger Hamiltonian as discussed next.

ii) Effect of strain on the bandstructure in a quantum well:

The nature of the strain in a quantum well is biaxial. In order for the strain to be accommodated coherently (no dislocations), the lattice constant of the well and barrier, in the direction parallel to the interface, must be equal i.e.

$$
a_{\| 1}=a_{\| 2}
$$

where the $a_{1}$ and $a_{2}$ are the lattice constants in the type 1 and 2 materials respectively. The perpendicular lattice constants are of course in general unequal. 
Growth of a material on a lattice mis-matched substrate will directly strain the parallel directions to match the substrate. The perpendicular direction however, is not directly strained and, therefore, is altered only by the Poisson effect. Thus for each material if

$$
a_{\| \text {strained }}=(1+\epsilon) a_{o}
$$

then we have

$$
a_{\perp \text { strained }}=(1-\sigma \epsilon) a_{0}
$$

where $\sigma$ is the appropriate Poisson constant and $\epsilon$ is the strain in the direction parallel to the interfaces.

The well material will then take on the lattice constant of the barrier material in the parallel directions, and its perpendicular lattice constant will be determined by equation (11). We emphasize that it is not certain that this simple picture of strain absorption is actually realized in nature. More experimental work on strained quantum wells, when compared with theory, may shed light on this issue.

For the calculations in this paper, we have used the thick barrier approximation to study the bandstructure of $\mathrm{GaAs} / \mathrm{AlGaAs}$, InGaAs/AlGaAs and GaAsP/AlGaAs systems. The thick barrier approximation predicts that in the second of these three systems, the well is under a compressive biaxial strain. In the last one, the well region is under tensile biaxial strain.

The effect of a biaxial strain (to first order) is to cause a splitting between the heavy-hole $(3 / 2,3 / 2)$ and light-hole $(3 / 2,1 / 2)$ states. The effects on the heavy and light-hole bandgaps are ${ }^{i U}$

$$
\begin{aligned}
& E_{g 3 / 2}=E_{g}+1 / 2 \delta E_{s h}-\delta E_{h y} \\
& E_{g 1 / 2}=E_{g}-1 / 2 \delta E_{s h}-\delta E_{h y}
\end{aligned}
$$

where.

$$
\begin{gathered}
\delta E_{s h}=-2 b\left[\left(C_{11}+2 C_{12}\right) / C_{11}\right] \epsilon \\
\delta E_{h y}=-2 a\left[\left(C_{11}-C_{12}\right) / C_{11}\right] \epsilon
\end{gathered}
$$

Here $\mathcal{C}_{11}, \mathcal{C}_{12}$ are force constants for the well material, $a$ and $b$ are the deformation potentials. For GaAs the shifts for the heavy and light-hole gaps are $-5.96 \epsilon$ and $-12.4 \epsilon^{10}$ Thus in presence of a biaxial tensile strain the light-hole is expected to be above the heavy-hole state while for compressive strain the reverse is expected.

To solve the electron and hole problems in the presence of biaxial strain one simply includes the splitting between the heavy and light-hole states in the diagonal terms of the Kohn Luttinger Hamiltonian. In presence of strain, since the bandgap changes, it is not evident if the discontinuity distribution will change. However, the changes are not found to have significant effects on the electron or hole problem.

\section{iii) Effect of Transverse Electric Field}

An electric field transverse to the quantum well in terfaces alters the general potential profile, $V(z)$. The new form which the profile takes on is $V(z)+e E z$. The effect of this, in general, is that the bound states become quasi-bound states which have a finite lifetime for tunneling out of the potential barrier. As long as the assumption that the envelope function decays rapidly in the barrier is valid, the method described above can be readily applied to study the electron or hole problem. A simple criteria to establish whether or not this assumption is valid is to increase the total region $L$ in which the problem is solved. A quasi-bound state should give eigenvalues and eigenfunctions that remain unaffected due to the variations in $\mathrm{L}$.

Note that there is a splitting of the hole states which arise due to the lack of symmetry in the (001) direction which lifts the Kramer's degeneracy. These splittings, away from the zone center have been experimentally observed for a GaAs/AlGaAs modulation doped structure since they cause a variation of the hole masses. The change in the masses causes the exciton binding energy to change as the electric field is increased and causes a broadening of the exciton transitions.

The dominant effect of the electric field is to squeeze the electron and hole states in opposite sides of the well. This reduces the overlap in the two states which affects the absorption coefficient. The intersubband separation is also effected.

\section{iv) The Exciton Problem}

The electron-hole pairs produced due to the photon absorption can form an exciton state due to the Coulombic interaction. The exciton problem can be expressed as $^{13,14}$

$$
\begin{aligned}
H= & -\frac{\hbar^{2}}{2 \mu}\left(\frac{1}{\rho} \frac{\partial}{\partial \rho} \rho \frac{\partial}{\partial \rho}+\frac{1}{\rho^{2}} \frac{\partial^{2}}{\partial \phi^{2}}\right)-\frac{\hbar^{2}}{2 m_{e}} \frac{\partial^{2}}{\partial z_{e}^{2}} \\
& -\frac{\hbar^{2}}{2 m_{h}} \frac{\partial^{2}}{\partial z_{h}^{2}}-\frac{e^{2}}{\epsilon\left|r_{e}-r_{h}\right|}+V_{e w}\left(z_{e}\right) \\
& +V_{h w}\left(z_{h}\right)+e E z_{e}-e E z_{h}
\end{aligned}
$$

where $m_{e}$ and $m_{h}$ are the effective mass of the conduction band electron and the valance band hole respectively. $\mu$ is the reduced mass corresponding to each electron and hole subband combination so that the first term describes the exciton internal kinetic energy in the plane perpendicular to the $z$ axis. We have assumed the same values for the static dielectric constant in the two semiconductors. The appropriate dielectric constant is taken to be $\kappa=\sqrt{\kappa_{\omega} \kappa_{b}}$, where $\kappa_{\omega}, \kappa_{b}$ are the dielectric constants of the well and barrier respectively. The potentials such as $V_{e w}\left(z_{e}\right)$ for electrons and $V_{h w}\left(z_{h}\right)$ for holes can be arbitrary confining potentials, which give 
rise to bound or quasi- bound states. The quantities $e E z_{e}$ and $e E z_{h}$ are the additional potential energy terms for electrons and holes due to the electric field.

To solve the exciton problem one essentially has to adjust the exciton envelop function to minimize the sum of the kinetic energy and the Coulombic potential energy terms. Unfortunately at present, there is no analytical or numerical terhnique to solve the exciton problem exactly and one has to rely on the variational approach. The equation for the exciton system can be written in the $\rho$-space or $\mathrm{k}$-space representation depending upon whether the variational approach is to be applied in the real or $\mathbf{k}$-space. From the variety of dispersion relations discussed for the hole states, it is clear that while some of the bands appear to be fairly parabolic (with an effective mass to be obtained by a numerical fit not the original Kohn-Luttinger bulk parameters), some of the states become highly non-parabolic. A clear example of this situation is the light-hole ground states (LH1) which display a negative effective mass at the zone center due to the very strong mixing with the heavy-hole states. For such states it is clear that one either has to solve the exciton problem explicitly in $\mathbf{k}$-space or a fit for the exciton kinetic energy of the form

$$
E_{K . E .}^{e x}\left(k_{\|}\right)=E^{e}\left(k_{\|}\right)+E^{h}\left(k_{\|}\right)
$$

with

$$
\begin{aligned}
& E^{e}\left(k_{\|}\right)=\frac{\hbar^{2}}{2 m_{e}^{*}} k_{\|}^{2} \\
& E^{h}\left(k_{\|}\right)=\alpha k_{\|}^{2}+\beta k_{\|}^{4}
\end{aligned}
$$

must be made. Here the parameters $\alpha$ and $\beta$ are to be determined numerically by fitting the hole dispersion relation. The hole dispersion relation is assumed to be isotropic to simplify the calculations.

A number of envelope function forms have been assumed by different workers ${ }^{11-14}$. These include exponentially falling functions (as for the hydrogen atom problem), gaussian functions, etc. A simple exponential envelope function is found to work quite well when the exciton energy is parabolic $(\beta=0)$. However, a Gaussian works better for non-parabolic bands. The variational calculation involves minimizing the energy

$$
E=\frac{\iiint \Psi^{*} H \Psi d z_{e} d z_{h} \rho d \rho}{\iiint \Psi^{*} \Psi d z_{e} d z_{h} \rho d \rho}
$$

as a function of the variables involved in the envelope function.

Once the excitons are formed in the quantum well due to photon absorption, and the electric field pulse causing the absorption is back to normal, it is important for Electro-Optical modulators that the space charge in the well be removed quickly. This is possible either by exciton recombination or the electron and hole tunneling out at high electric fields.

An important issue for optical modulators and detectors based on quantum wells is the inhomogeneous broadening caused by structural fluctuations in the quantum well system. These fluctations can be loosely categorized as interface roughness effects and alloy disorder effects. The effect of these fluctuations is to broaden the exciton transitions and since broadening can seriously reduce the on/off intensity ratio, i.e.the modulation depth, it is important to understand the extent of the broadening caused by structural fluctuations.

The general method used to treat the inhomogeneous line broadening is discussed in reference 15,16 , and 17 so we will only briefly discuss the main broadening mechanisms.

The dominant exciton broadening mechanisms are due to:

i) phonon broadening, which has the form ${ }^{18}$

$$
\sigma_{t o t}=\sigma_{o}+\alpha T+\frac{\beta}{\exp \left(\frac{\hbar w_{o}}{k_{B} T}\right)-1}
$$

where $\sigma_{o}, \alpha$ and $\beta$ are temperature-independent and $w_{o}$ is the polar optical phonon frequency. The phonon contribution to the exciton linewidth is less than $0.5 \mathrm{meV}$ at $77 \mathrm{~K}$ and $\sim 3 \mathrm{meV}$ at $300 \mathrm{~K}$.

ii) tunneling of electrons and holes and the conse quent broadening due to finite lifetime. This mcchanism does not contribute much to the exciton linewidth except at very high electric fields $(E>75 \mathrm{kV} / \mathrm{cm}){ }^{16}$

iii) lifting of Kramer's degeneracy. This is due to the splitting of the doubly degenerate hole states in presence of an electric field. This causes a broadening since the exciton binding energy splits slightly $(\sim 1 \mathrm{meV}$ at $E=$ $50 \mathrm{kV} / \mathrm{cm}$ ).

iv) interface quality and well-towell size fluctuations. ${ }^{16}$ This can be a very important source of broadening and is controlled by the quantity $\partial \mathrm{E}_{e x} / \partial \mathrm{W} \mid W_{o}$, which is the variation of the exciton transition energy due to well width fluctuation at well width $W_{n}$. This quantity increases rapidly with applied electric field so that this mechanism becomes extremely important at high fields. In addition, alloy broadening can also be an important broadening mechanism, particularly for systems where the well region is an alloy.

In this paper, we will use the low temperature exciton linewidth to estimate the quality of the material and to fit the data. The theoretical results presented for systems where we do not currently have experimental results are for a one monolayer well size fluctuation. 
v) Polarization Dependence of Optical Absorption in Quantum Wells

Once the exciton wavefunction, energy and the electron and hole dispersion relations have been determined in the quantum well, it is straightforward to determine the absorption coefficients for the excitonic and band-toband transitions. Using first order perturbation theory the absorption coefficients for band-to-band transition is given from the Fermi's golden rule as ${ }^{19}$

$$
\begin{aligned}
\alpha(\hbar \omega ; \hat{\epsilon})= & \frac{4 \pi^{2} e^{2} \hbar}{\eta c m^{2} \hbar \omega} \cdot \sum_{k_{\|}} \sum_{n, m}\left|\hat{\epsilon} \cdot p_{n m}\left(k_{\|}\right)\right|^{2} \\
& . \delta\left(E_{m}^{h}\left(k_{\|}\right)-E_{n}^{e}\left(k_{\|}\right)+\hbar \omega\right)
\end{aligned}
$$

where $\eta$ is the refractive index of the semiconductor, $\omega$ the photon frequency, $\hat{\epsilon}$ polarization vector of the radiation, $m, n$ the hole and electron subband indices. The optical matrix is given by,

$$
\mathbf{p}_{n m}\left(k_{\|}\right)=\left\langle\Psi_{m}^{h}\left(k_{\|}\right)|\mathbf{p}| \Psi_{n}^{e}\left(k_{\|}\right)\right\rangle
$$

where $\Psi_{m}^{h}\left(k_{\|}\right)$and $\Psi_{n}^{e}\left(k_{\|}\right)$are the hole and electron wave functions determined previously. A broadening function is usually introduced to replace the Dirac $\delta$-function to introduce the broadening due to various mechanisms.

The optical matrix elements are given by

$$
\mathrm{p}_{n m}\left(k_{\|}\right)=\sum_{\nu, \mu} \int d r L_{0}^{\nu^{\prime *}}(r) \mathrm{p} U_{0}^{\mu}(r) \int d z f_{n}^{\mu}(z) g_{m}^{\nu *}\left(k_{\| l}, z\right)
$$

The first part of the matrix elements is determined from the Bloch functions of the s-type spin $\frac{1}{2}$ conduction band states and $\mathrm{p}$-type angular momentum $\frac{3}{2}$ valence band states. We have already discussed the relations between the hole angular momentum states and the $p_{x}, p_{y}, p_{z}$ states. The relevant matrix elements then become,

$$
\begin{aligned}
& \left\langle \pm \frac{3}{2}\left|p_{x}\right| \pm \frac{1}{2}\right\rangle=\frac{1}{\sqrt{2}}\left\langle x\left|p_{x}\right| \mu\right\rangle \\
& \left\langle \pm \frac{1}{2}\left|p_{x}\right| \mp \frac{1}{2}\right\rangle=\frac{1}{\sqrt{6}}\left\langle x\left|p_{x}\right| \mu\right\rangle \\
& \left\langle \pm \frac{1}{2}\left|p_{z}\right| \pm \frac{1}{2}\right\rangle=\frac{2}{\sqrt{6}}\left\langle x\left|p_{x}\right| \mu\right\rangle
\end{aligned}
$$

The matrix element $\left\langle x\left|p_{x}\right| \mu\right\rangle$ between the atomic like states is dependent on the semiconductor material under consideration. Values for some semiconductors are tabulated by Lawaet $z^{20}$. The matrix elements $\mathbf{p}_{\pi m}\left(k_{\|}\right)$vary with $k$ and also have strong polarization dependences as will be shown later. The band-to-band absorption is then proportional to

$$
\alpha(\hbar \omega ; \hat{\epsilon}) \propto \sum_{n, m}\left|\hat{\epsilon} \cdot p_{n m}\left(k_{\|}\right)\right|^{2} \delta_{n m} \rho_{n m}(\hbar \omega)
$$

where $\rho_{n m}(\hbar \omega)$ is the joint density of the states of the electron-hole system. Factor $\left|\hat{\epsilon} \cdot p_{n m}\left(k_{\|}\right)\right|^{2}$ is a weighted matrix element for electron $\mathrm{n}$ band and hole $\mathrm{m}$ band transition, which has anistropic nature with respect to polarization. For simple parabolic bands

$$
\rho_{n m}(\hbar \omega)=\sum_{n m} \frac{\mu_{n m}}{\pi \hbar^{2}} \Theta_{n m}(\hbar \omega)
$$

where $\mu_{n m}$ is the reduced mass of electron subband $n$ and hole subband $m$, and $\Theta_{n m}(\hbar \omega)$ is a step function but for non-parabolic bands one has to explicitly calculate the density of states. For example, for LH1 transitions it is clear from earlier discussions that the step density function is not valid. Here, to include the band-to-band transition broadening effect we made a convolution of broadening function and $\Theta_{n m}(\hbar \omega)$.

Fur the calculation of the excitonic absorption cuefficient one cannot treat $p_{n m}\left(k_{\|}\right)$to be independent of $k_{\|}$. However, it is found to be reasonable to take $p_{n m}\left(k_{\|}\right)$to independent of the direction of $p_{n m}\left(k_{\|}\right)^{10}$. The exciton absorption coefficent is given by

$$
\alpha_{n m}=\frac{4 \pi^{2} e^{2} \hbar}{\eta m_{0} c W} \frac{1}{\hbar \omega}\left|\sum_{k_{\|}} G_{n m}\left(k_{\|}\right) \hat{\epsilon} \cdot p_{n m}\left(k_{\|}\right)\right|^{2} \delta\left(\hbar \omega-E_{n m}\right)
$$

Here the $G_{n m}\left(k_{i \mid}\right)$ are the Fourier components of the exciton envelope function and are determined by the solution of the exciton problem. $W$ is effective well width of QW structure. Once again the Dirac $\delta$-function has to be replaced by the broadening function since there is always a certain amount of linewidth in the exciton transition. If the width is due to inhomogeneous broadening as discussed in the previous section, the appropriate replacement is

$$
\delta\left(\hbar \omega-E_{n m}\right)=\frac{1}{\sqrt{1.44 \pi} \sigma} \exp \left(\frac{-\left(\hbar \omega-E_{n m}\right)^{2}}{1.44 \sigma^{2}}\right)
$$

Here $\sigma$ is the linewidth (corresponding to the half-width at half-maximum). The inhomogeneous broadening is a sensitive function of the transverse electric field and must be incorporated in the calculation for correct results.

The polarization dependence of the absorption becomes apparent once the nature of the heavy-hole and light-hole states is expressed in terms of the p-type orbitals. This relationship is given by the following set of equations:

$$
\begin{aligned}
H H: \psi_{\frac{3}{2}, \frac{2}{2}} & =\phi_{11} \uparrow \\
L H: \psi_{\frac{3}{2}, \frac{1}{2}} & =\frac{1}{\sqrt{3}} \phi_{11} \downarrow+\frac{\sqrt{2}}{\sqrt{3}} \phi_{10} \uparrow \\
L H: \psi_{\frac{3}{2},-\frac{1}{2}} & =\frac{\sqrt{2}}{\sqrt{3}} \phi_{10} \downarrow+\frac{1}{\sqrt{3}} \phi_{1-1} \uparrow \\
H H: \psi_{\frac{3}{2},-\frac{3}{2}} & =\phi_{1-1} \downarrow
\end{aligned}
$$



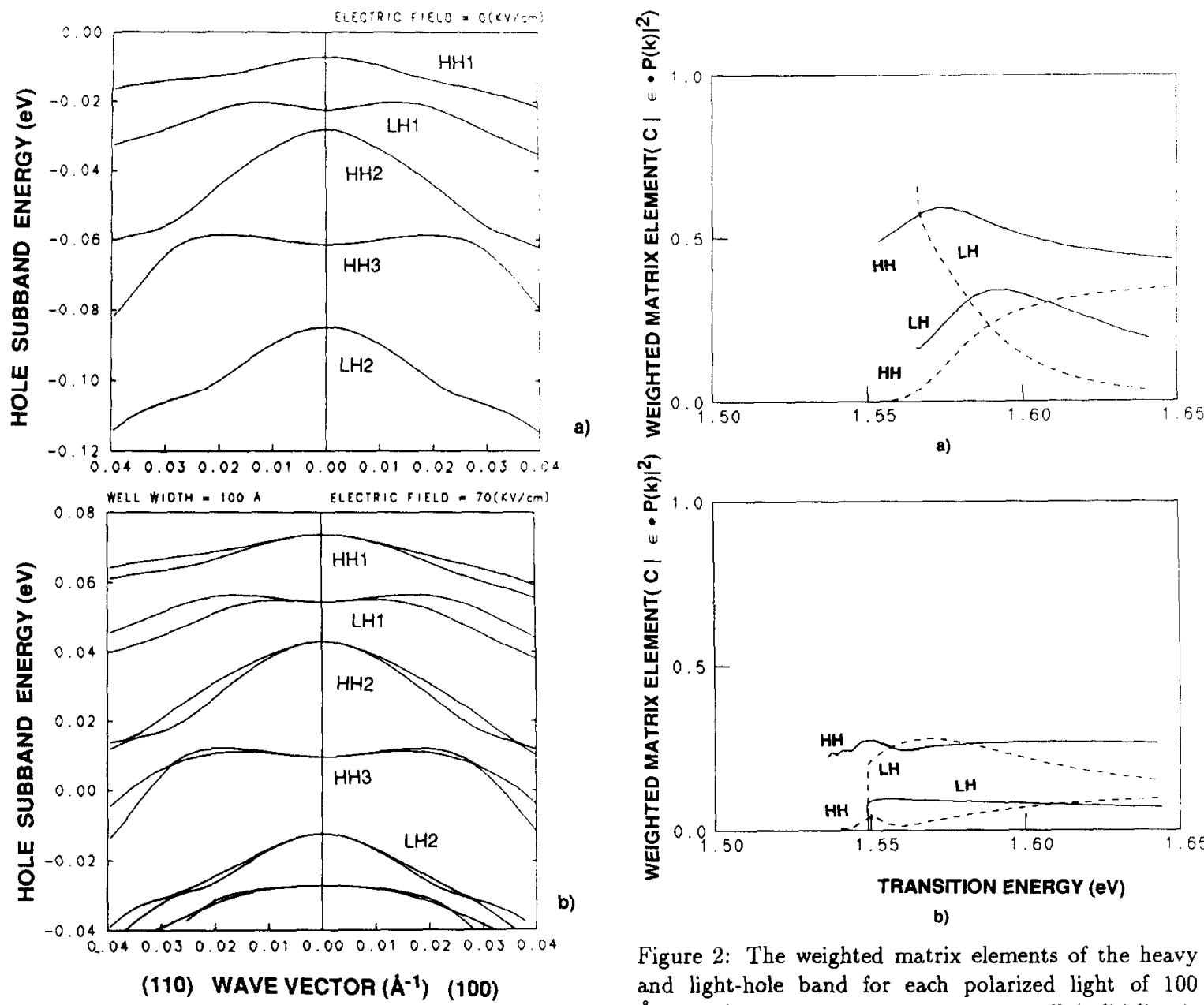

b)

Figure 2: The weighted matrix elements of the heavy and light-hole band for each polarized light of 100 AGaAs / $/ \mathrm{Al}_{0.3} \mathrm{Ga}_{0.7} \mathrm{As}$ multi-quantum well (solid line is

Figure 1: Hole dispersion of $100 \AA \mathrm{GaAs} / \mathrm{Al}_{0.3} \mathrm{Ga}_{0.7} \mathrm{As}$ multi-quantum well a) $0 \mathrm{KV} / \mathrm{cm}$ case, b) $70 \mathrm{KV} / \mathrm{cm}$ case.

Here $\phi_{i, j}$ are the orbital angular momentum eigenfunctions with angular momentum ' $i$ ' and projection ' $j$ ' and are related to the $\mathrm{p}$ orbitals by:

$$
\begin{aligned}
& \mid p_{x} \uparrow>=\frac{1}{\sqrt{2}}\left(\phi_{11}+\phi_{1-1}\right) \uparrow \\
& \mid p_{y} \uparrow>=\frac{-i}{\sqrt{2}}\left(\phi_{11}-\phi_{1-1}\right) \uparrow \\
& \mid p_{z} \downarrow>=\phi_{10} \downarrow
\end{aligned}
$$

It is clear for example that a pure $\mid \frac{3}{2}, \pm \frac{3}{2}>$ state is made up of $p_{x}$ and $p_{y}$ components and therefore if $\hat{\epsilon}$ is along the $\mathrm{z}$ - axis (growth direction), the absorption will vanish. However, it must be pointed out that states are

pure only at the zone center in the Kohn- Luttinger formalism and there is a strong mixing of states away from $k=0$. These effects are examined in the next section.

\section{RESULTS}

The room temperature excitonic resonance in lattice matched GaAs/AlGaAs MQW structure has been exploited for high speed electro - optical modulators and optical switches. An additional interesting effect which can be used for optical modulation and switching devices is the highly anisotropic absorption for light propagating parallel to the plane of the layers. Recently, efforts are also being directed towards controlling the absorption profile by introducing strain in the well region. In this section we will show the polarization effect in lattice 


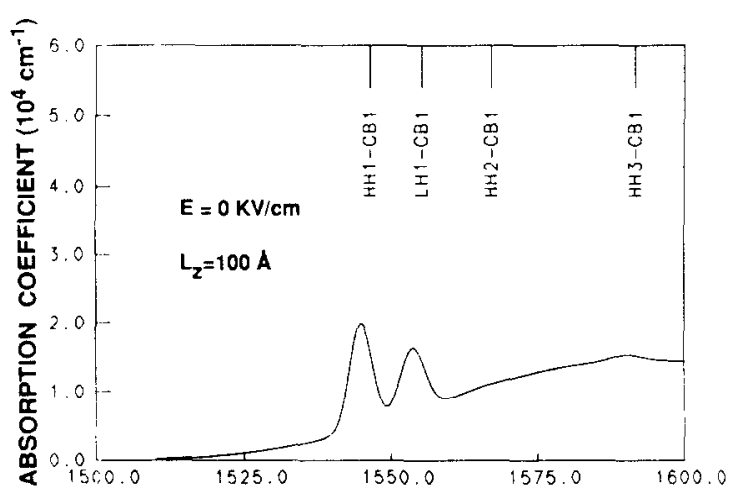

a)

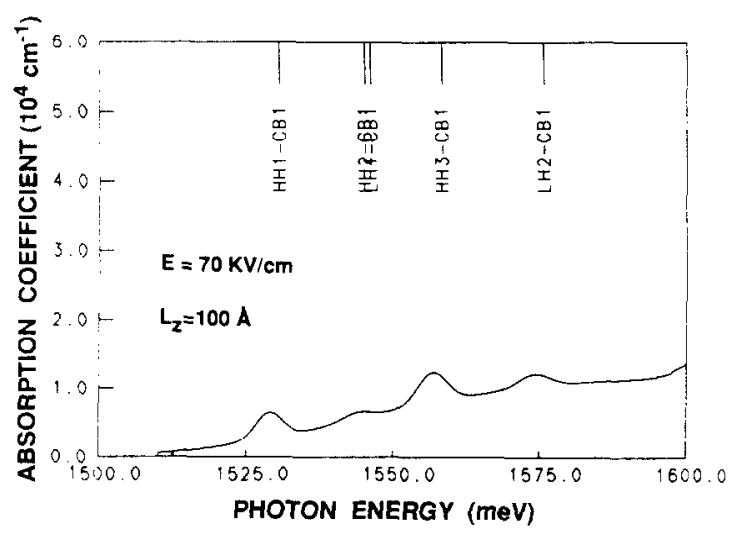

b)

Figure 3: $x-y$ polarized light absorption of GaAs $/ \mathrm{Al}_{0.3} \mathrm{Ga}_{0.7} \mathrm{As}$ multi quantum well structure in wave guide type application a) $0 \mathrm{KV} / \mathrm{cm}$ case, b) $70 \mathrm{KV} / \mathrm{cm}$ case.

matched and strained systems with and without electric field. To explain this anisotropic nature, we show the band structures, the matrix elements of heavy-hole and light-hole transitions and absorption profiles for both parallel and perpendicular polarized light.

Figure 1 a) shows the hole dispersion relation in a 100 $\AA \mathrm{GaAs} / \mathrm{Al}_{0.3} \mathrm{Ga}_{0.7}$ As multi-quantum well without electric field. The results show strong nonparabolic band structures due to heavy-hole and light-hole band mixing. Figure $1 \mathrm{~b}$ ) shows the band structure in presence of a $70 \mathrm{kV} / \mathrm{cm}$ transverse electric field. Note that due to the lifting of Kramer's degeneracy each hole band is no longer doublely degenerate. We investigate the weighted matrix elements $\left|\epsilon \cdot P\left(k_{\|}\right)\right|^{2}$ for heavy hole and light-hole bands beacuse this factor is directly related to excitonic absorption as well as interband absorption. Figure 2 a) shows the weighted matrix elements oi the heavy and
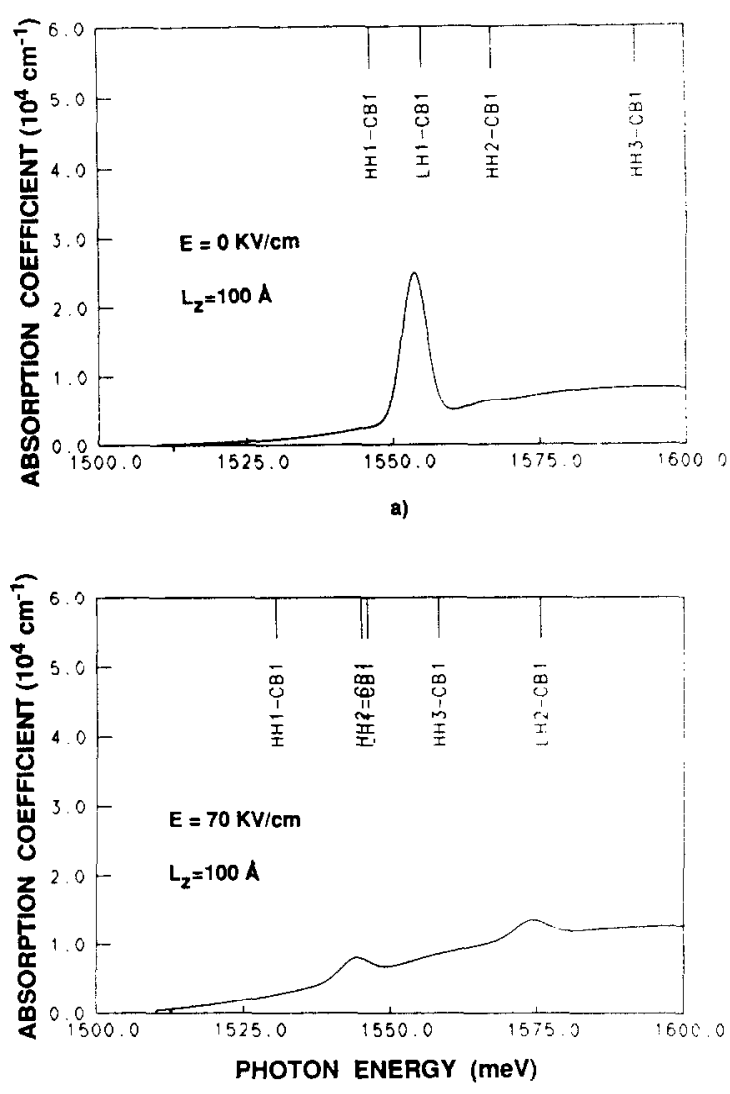

b)

Figure 4: $\mathrm{z}$ polarized light absorption of GaAs $/ \mathrm{Al}_{0.3} \mathrm{Ga}_{0.7} \mathrm{As}$ multi quantum well structure in wave guide type application a) $0 \mathrm{KV} / \mathrm{cm}$ case, b) $70 \mathrm{KV} / \mathrm{cm}$ case.

light-hole band for the two polarizations (solid line is for $\mathrm{x}-\mathrm{y}$ polarized light and dotted line for $\mathrm{z}$ polarized light). Note that the states are pure only at zone center and the weighted matrix elements change with respect to energy. These weighted matrix elements shown in the figures are nomalized by the factor $\left|\left\langle x\left|p_{x}\right| \mu\right\rangle\right|^{2}$. This complex variation of these factors is due to hole band mixing near the zone center. Note that if there is no hole band mixing, excitonic and interband absorption of heavy-hole for the $z$ polarized light should be absolutely zero. But actually, away from the zone center, light hole nature exist even in the heavy-hole band because of hole band mixing. Thus we see a small heavyhole transition even in $z$ polarized light absorption spectra. Figure $2 \mathrm{~b}$ ) gives the variation of these factors at $70 \mathrm{kV} / \mathrm{cm}$. Comparing figure $2 \mathrm{a}$ ) and figure $2 \mathrm{~b}$ ), we observe that band mixing is reduced by applying electric 

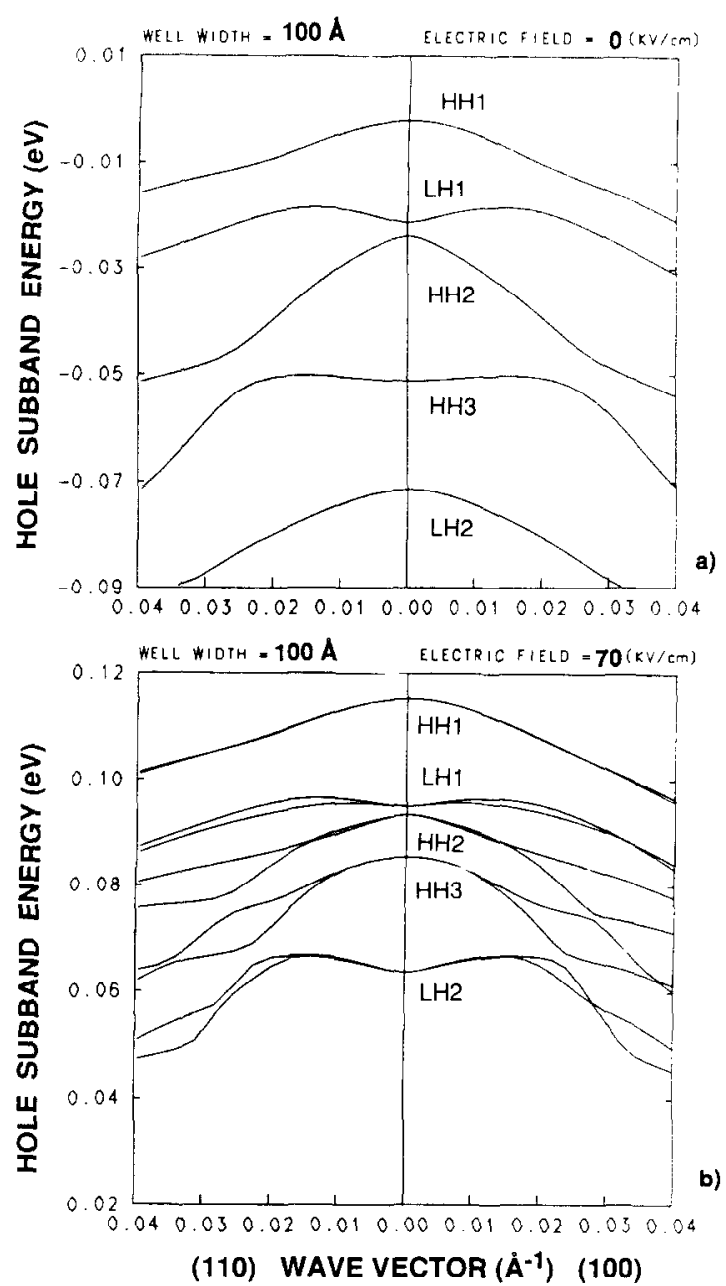

Figure 5: Hole dispersion of $100 \AA \operatorname{In}_{0.02} \mathrm{Ga}_{0.98} \mathrm{As}$ $/ \mathrm{AI}_{0.3} \mathrm{Ga}_{0.7} \mathrm{As}$ multi-quantum well a) $0 \mathrm{KV} / \mathrm{cm}$ case, b) $70 \mathrm{KV} / \mathrm{cm}$ case.

field, and thus we expect not only differences in interband absorption with respect to the polarization of the incident light, but also that this difference is reduced by applying transverse electric field. In figure 3 and 4 we have shown the polarization dependence of the absorption of a GaAs $/ \mathrm{Al}_{0.3} \mathrm{Ga}_{0.7}$ As multi quantum well structure in wave guide type application. Figure 3 shows the absorption modulation for the $x-y$ polarized light due to a transverse electric field. Figure 3 a) and Figure 3 b) are absorption profiles for the zero electric field case and the $70 \mathrm{KV} / \mathrm{cm}$ case, respectively. Figure 4 shows
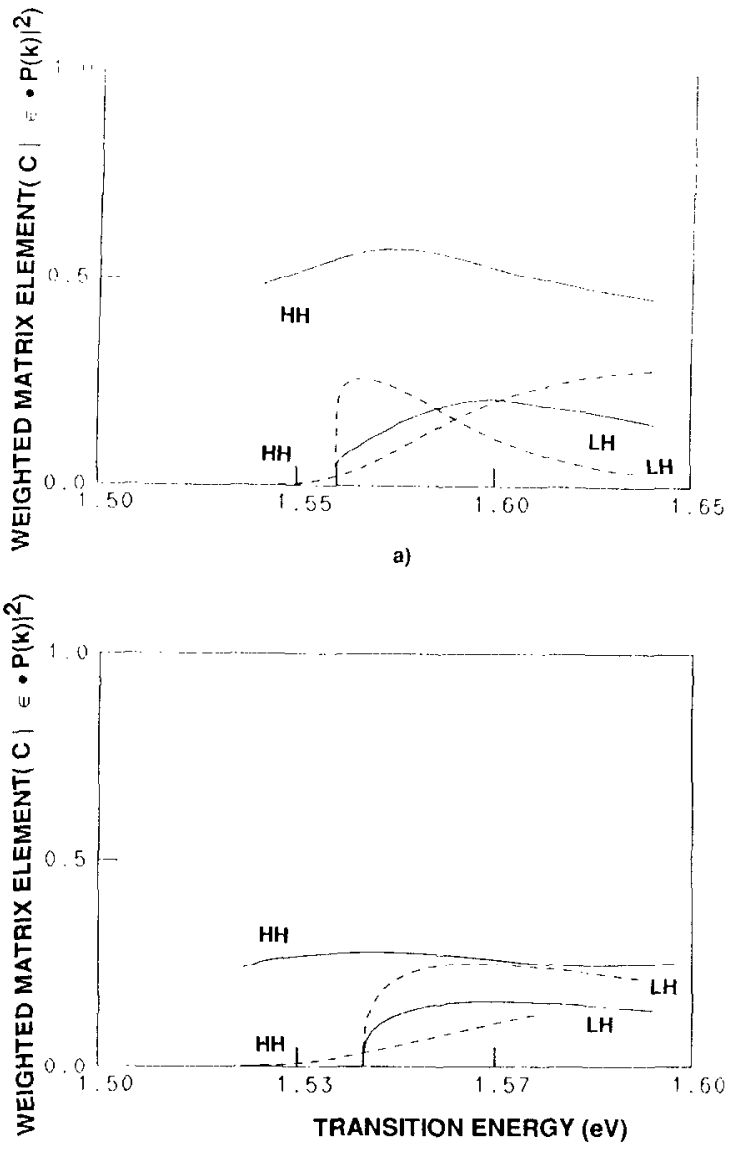

b)

Figure 6: The weighted matrix elements of the heavy and light-hole band for each polarized light of 100 $\AA \mathrm{In}_{0.02} \mathrm{Ga}_{0.98} \mathrm{As} / \mathrm{Al}_{0.3} \mathrm{Ga}_{0.7}$ As multi-quantum well (solid line is for $x-y$ polarized light and dotted line for $z$ polarized light) a) $0 \mathrm{KV} / \mathrm{cm}$ case, b) $70 \mathrm{KV} / \mathrm{cm}$ case.

the absorption coefficient of this quantum well with and without electric field for $z$ polarized light case. Here we have introduced the line broadening effect by assuming a 1 monolayer well-to-well size fuctuation and constant 3 $\mathrm{meV}$ broadening corresponding to homogeneous broadening at room temperature. The results of figure 3 and 4 compare quite well with the published experimental results of Wiener et. al. ${ }^{21}$

In figure 5 we show the hole dispersions of 100 $\AA \mathrm{In}_{0.02} \mathrm{Ga}_{0.98} \mathrm{As} / \mathrm{Al}_{0.3} \mathrm{Ga}_{0.7}$ As quantum well structure, which have compressive strain in the well region. Heavy 


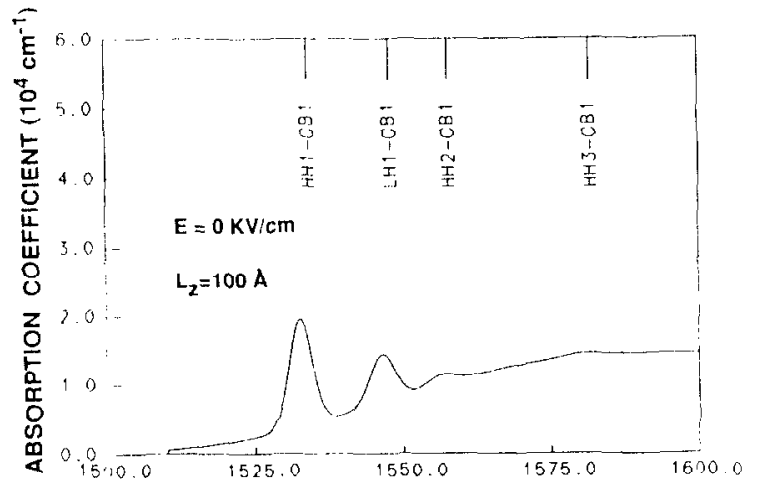

a)

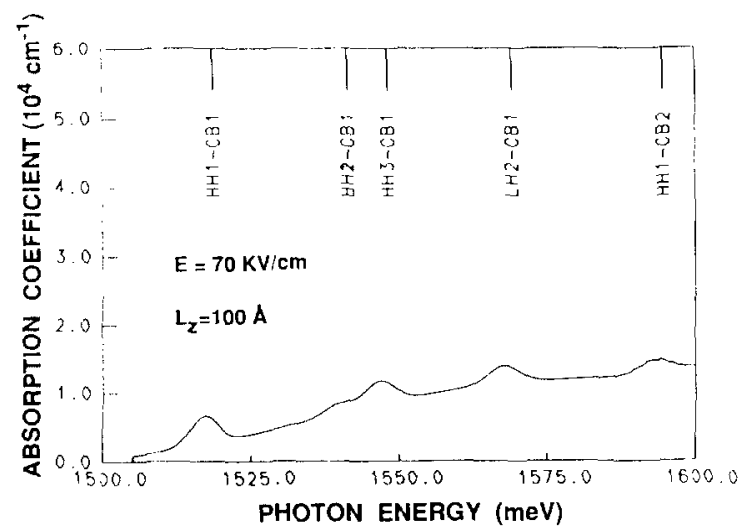

b)

Figure 7: $x-y$ polarized light absorption of $\operatorname{In}_{0.02} \mathrm{Ga}_{0.98} \mathrm{As}$ $/ \mathrm{Al}_{0.3} \mathrm{Ga}_{0.7} \mathrm{As}$ multi quantum well structure in wave guide type application a) $0 \mathrm{KV} / \mathrm{cm}$ case, b) $70 \mathrm{KV} / \mathrm{cm}$ rase.

hole and light-hole band are seperated to some extent by the strain in the well. Figure 6 shows the weighted matrix elements of the heavy and light-hole band for each polarized light with and without electric field. Once again from comparision of figure 6 a) and b), we observe that the band mixing is reduced with electric field. Also from the comparision of figure 6 a) and figure 2 a) we find that compressive strain also reduce the band mixing by separating the $\mathrm{HH}$ and $\mathrm{LH}$ states. Figure 7 and 8 are the absorption coefficients of this quantum well, with and without electric field, for each light polarization direction.
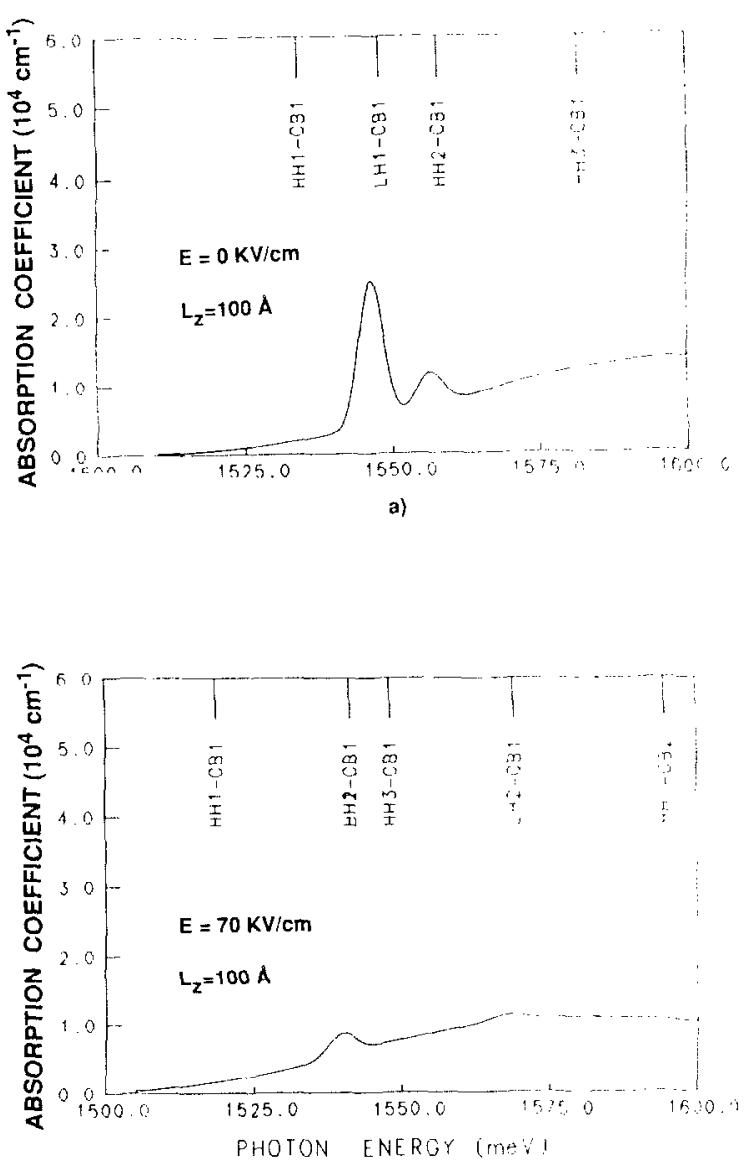

b)

Figure 8: $z$ polarized light absorption of $\operatorname{In}_{0.02} \mathrm{Ga}_{0.98}$. As $/ \mathrm{Al}_{0.3} \mathrm{Ga}_{0.7} \mathrm{As}$ multi quantum well structure in wave guide type application a) $0 \mathrm{KV} / \mathrm{cm}$ case b) $70 \mathrm{KV} / \mathrm{cm}$ case.

Figure 9 shows the band structure of 100 $\AA_{G a A s}$.947 $_{3} \mathrm{P}_{0.053} / \mathrm{Al}_{0.3} \mathrm{Ga}_{0.3} \mathrm{As}$ with and without electric field. Figure 9 b) is for an electric field of $70 \mathrm{KV} / \mathrm{cm}$. This system has a tensile strain in the well region. Ir this system we have obtained a coincidence of heavy-hole and light-hole peak by careful choice of Indium composition. Figure 10 shows the weighted matrix elements of the heavy and light hole band for each light polarization direction, and shows the strong band mixing at zone center when $\mathrm{HH}$ and $\mathrm{LH}$ are merged at zero electrir field. If electric field is applied to this system the heavy. hole and light-hole band are no longer merged and band 

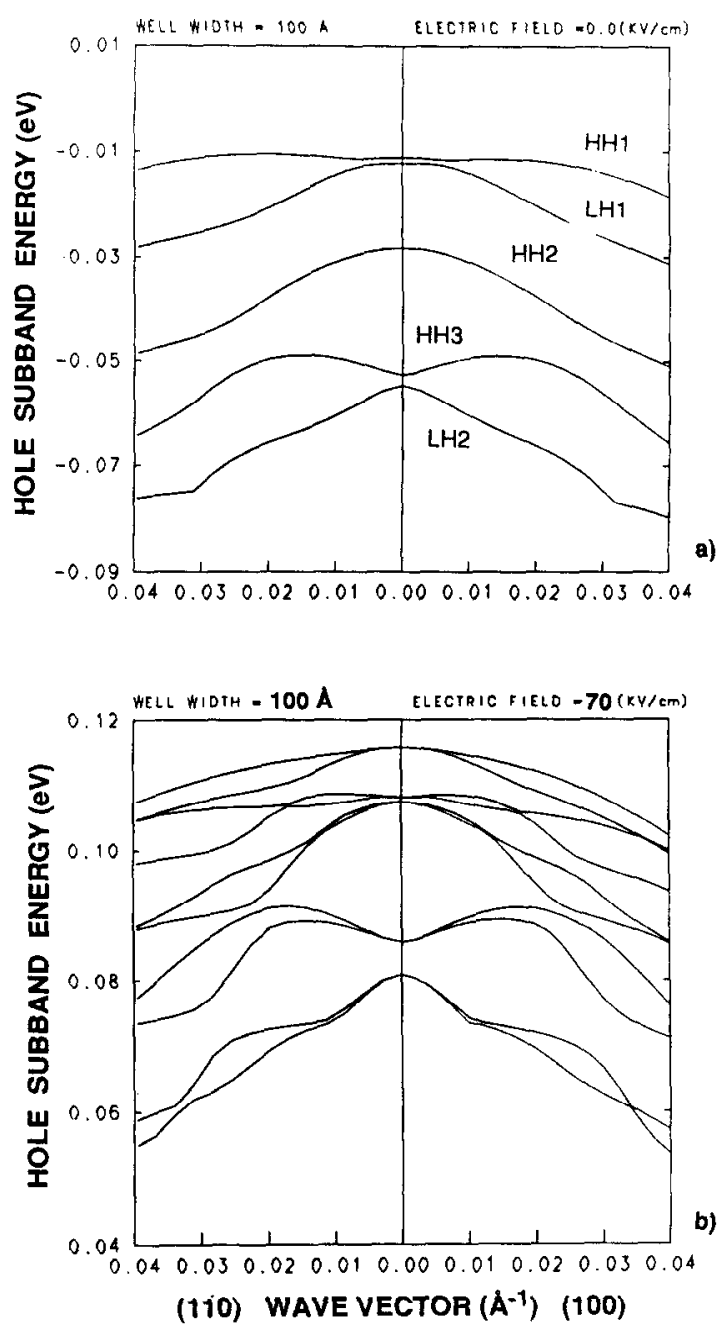

Figure 9: Hole dispersion of $100 \AA \mathrm{GaAs}_{0.947} \mathrm{P}_{0.053}$ (Al $\mathrm{l}_{0.3} \mathrm{Ga}_{0.7} \mathrm{As}$ multi-quantum well a) $0 \mathrm{KV} / \mathrm{cm}$ case, b) $70 \mathrm{KV} / \mathrm{cm}$ case.

mixing is reduced as shown in Figure $10 \mathrm{~b}$ ). Here we point out that as the HH and LH bands come closer the band mixing increases. The hole band seperation can be tailored by both applying electric field and introducing strain. In figure 11 a) and $11 \mathrm{~b}$ ) we show the absorption spectra for this system in absence and presence of the electric field for the $\mathrm{x}-\mathrm{y}$ polarization. In figure $12 \mathrm{a}$ ) and $12 \mathrm{~b}$ ) the same are plotted for the z-polarization. In this case the $\mathrm{HH}$ and $\mathrm{LH}$ peaks are the same energy at zero electric field and the effect of the strong band mixing is evident in the polarization dependence of the
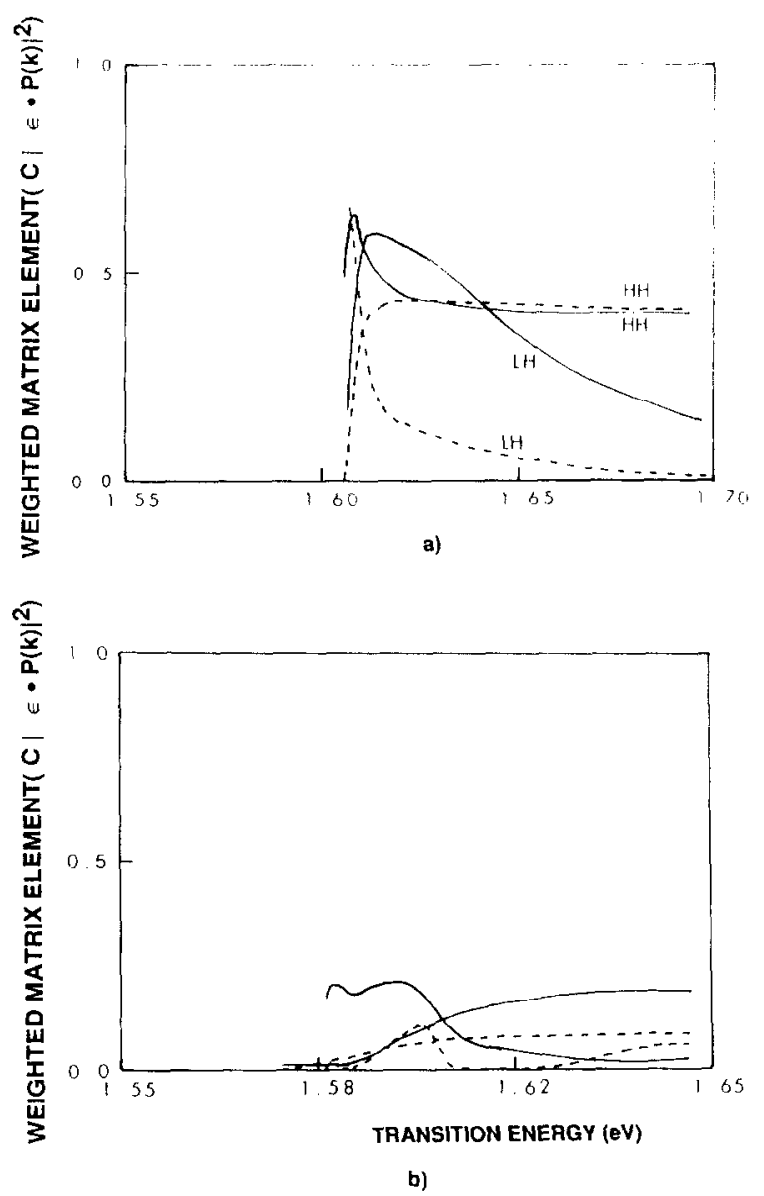

Figure 10: The weighted matrix elements of the heavy and light-hole band for each polarized light of $100 \AA \mathrm{AaAs}_{0.947} \mathrm{P}_{0.053} / \mathrm{Al}_{0.3} \mathrm{Ga}_{0.7} \mathrm{As}$ multi-quantum well (solid line is for $\mathrm{x}$-y polarized light and dotted line for $\mathrm{z}$ polarized light) a) $0 \mathrm{KV} / \mathrm{cm}$ case, b) $70 \mathrm{KV} / \mathrm{cm}$ case.

absorption. At present there are no published experimental polarization dependences of absorption in such strained systems and it is hoped the results shown here will motivate studies in this important area.

\section{CONCLUSION}

In this paper we have presented polarization depen dent optical absorption for excitonic and interband transitions in lattice matched (GaAs/AlGaAs) and strained 


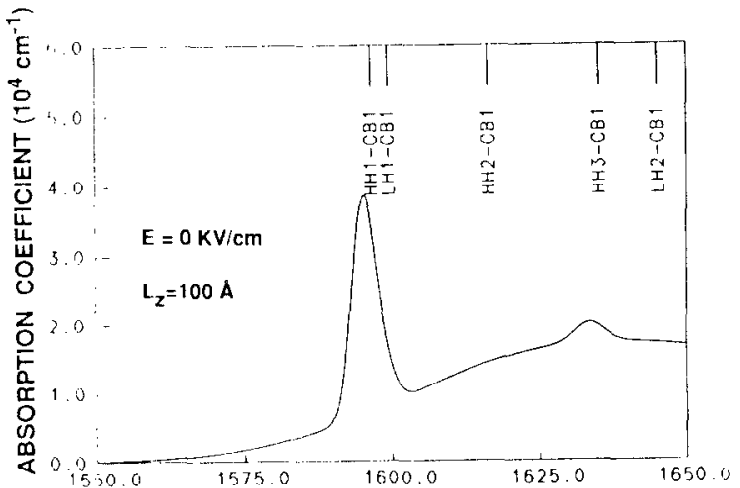

a)

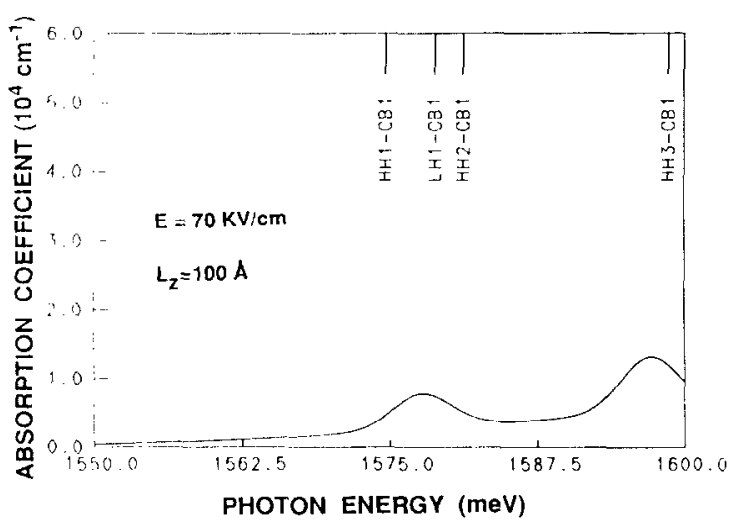

b)

Figure 11: $x+y$ polarized light absorption of 100 $\not \mathrm{GaAs}_{0.947} \mathrm{P}_{0.053} / \mathrm{Al}_{0.3} \mathrm{Ga}_{0.7} \mathrm{As}$ multi quantum well structure in wave guide type application a) $0 \mathrm{KV} / \mathrm{cm}$ (ase, b) $70 \mathrm{KV} / \mathrm{cm}$ case.

(biaxial tensile strain - GaAsP/AlGaAs; biaxial compressive strain - InGaAs/AlGaAs) multi-quantum weli structures in presence of transverse electric field. We have used the Kohn-Luttinger Hamiltonian to describe the top of the valence band and applied the eigenvalue method to calculate energy bands and wave functions. We have studied the effect of polarization on heavy-hole and light-hole bands including hole band mixing. We find that we can tailor the anisotropic absorption by controlling band mixing. The band mixing can be controlled either by applying electric field and/or by introducing strain in the well region. This anisotropic nature of absorption in the quantum well is quite strong and can be used in optical modulation or switching. The modulation by electic field and strain is also expected
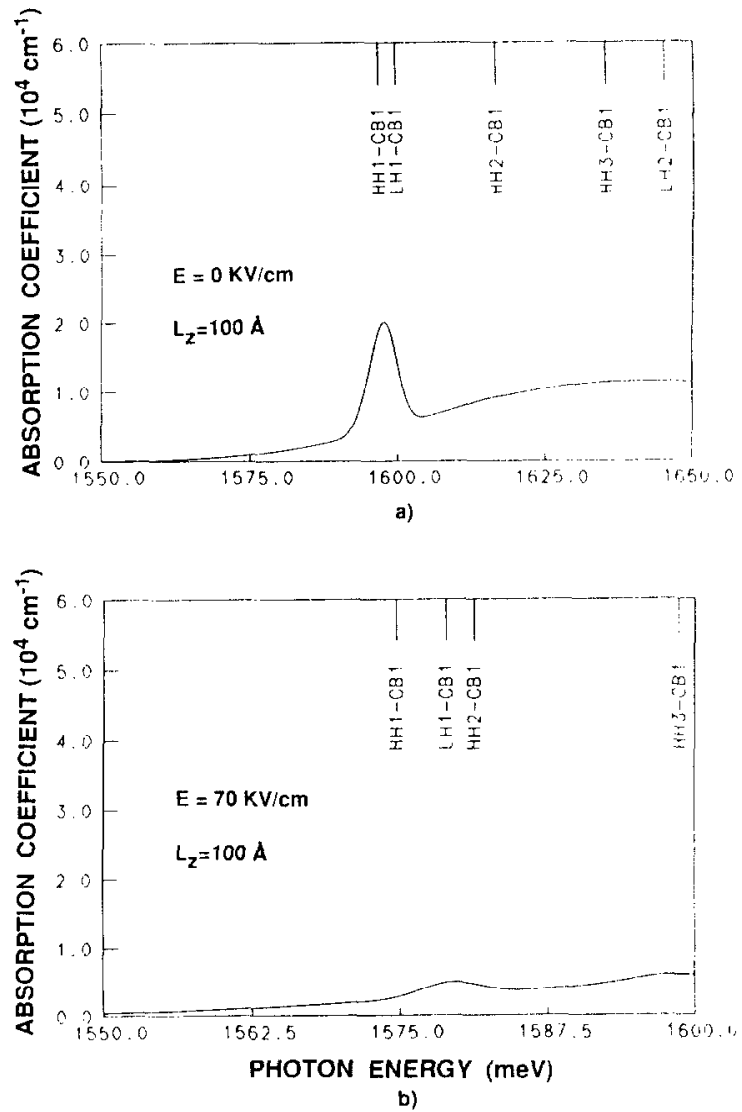

Figure 12: $z$ polarized light absorption of 100 $\AA \mathrm{GaAs}_{0.947} \mathrm{P}_{0.053} / \mathrm{Al}_{0.3} \mathrm{Ga}_{0.7} \mathrm{As}$ multi quantum well structure in wave guide type application a) $0 \mathrm{KV} / \mathrm{cm}$ case, b) $70 \mathrm{KV} / \mathrm{cm}$ case.

to be very useful in polarization sensitive optical device application.

Acknowledgement- The authors gratefully acknowledge valuable discussions with Prof. P.K. Bhattacharya. This work was funded by the NSF Lightwave Technology program under grant ECE 8610803.

\section{References:}

1. G. Bastard, Physics Review B, Vol. 26. 197 (1982)

2. J.S. Weiner, D.S. Chemla, D.A.B. Miller, T.H. Wood, D. Sivco and A.Y. Cho, Applied Physics Letter Vol 46, 619(1985) 
3. D.A.B. Miller, D.S. Chemla, T.C. Damen, A.C. Gossard, W. Wiegman, T.H. Wood, and C.A. Burrus, Physics Review B, Vol 32, 1043(1985)

4. W.D. Goodhue, B.E. Burke, K.B. Nichols, G.M. Metze, and G.D. Johnson, presented at the 6th MBE Workshop, Minneapolis, MN, Aug.(1985)

5. T. H. Wood, C. A. Burrus, D. A. B. Miller, D. S. Chemla, T. C. Damen, A. C. Gossard, and W. Wiegman, Applied Physics Letter, Vol.44, 16 (1984).

6. K. Wakita, Y. Kawamura, Y. Yoshikuni and H. Asahi, IEEE, Vol. QE-22, No 9, 1831(1986)

7. J. M. Luttinger and W. Kohn, Physics Review Vol. 97,869 (1955).

8. G.E. Pikus and G.L. Bir, Sov. Phys. Solid State 1, 1502(1959)

9. J. N. Schulman and Y. C. Chang, Physics Review B Vol. 24, 4445 (1981).

10. H. Kato, N. Iguchi, S. Chika, M. Nakayama and N. Sano, Journal of Applied Physics, Vol. 52(2), 588(1986)

11. G. D. Sanders and Y. C. Chang, Physics Review B Vol. 32, 5517 (1985).
12. E. Bangerk and G. Landwehr, Superlattices and Microstructures, Vol. 1, 363 (1985).

13. R. L. Greene and K. K. Bajaj, Journal of Vaccum Science of Technology Vol. B1(2), 391 (1985).

14. G. Bastard, Journal of Luminescence Vol. 30,488 (1985).

15. J. Singh and K. K. Bajaj, Applied Physics Letter Vol. 48, 1077 (1986).

16. S. Hong and J. Singh, Applied Physics Letter Vol. 49, 331 (1986).

17. S. Hong and J. Singh, Journal of Applied Physics to appear in Sept. 1, 1987 issue.

18. J. Lee, E. S. Koteles, and M. O. Vassell, Physics Review B Vol. 33, 5512 (1986).

19. See for example, F. Bassani and G. P. Parravicini, in "Electronic State and Optical Properties in Solids", Pergamon Press, New York 1975.

20. P. Lawaetz, Physics Review B Vol. 4, 3460 (1971).

21. J. S. Weiner, D. S. Chemla, and D. A. B. Miller, Applied Physics Letter, Vol 47, 664(1985) 